\title{
The Influence of Two-way Conflicts of Medical Staff work-families on Burnout
}

\author{
Sun Pinyi* \\ Human Resource Management \\ Zhejiang Institute of Technology \\ Hangzhou, Zhejiang \\ 545049820@qq.com
}

\author{
Wang Ping \\ Human Resource Management \\ Zhejiang Institute of Technology \\ Hangzhou, Zhejiang \\ wp190923@163.com
}

\begin{abstract}
The long working hours and high-intensity work pressure of medical staff cause conflicts between their family and work roles, a high level of burnout, and various psychological and physical problems, which are urgently needed to be resolved. Therefore, with the medical staff as the research object, the following conclusions were drawn through questionnaire distribution and statistical analysis of data: Medical staff have more obvious two-way conflicts and job burnout of work-families, and there are different degrees of differences in the work department, the number of children, and academic qualifications. In addition to family-work conflicts for emotional exhaustion, there is a significant positive correlation between two-way conflicts between work families and job burnout and their dimensions and the two-way conflict of work-families has a significant positive impact and a significant positive predictive effect on burnout. Based on the conclusions of this study, it is proposed from the three perspectives of hospital administrators, medical staff and society to reduce the two-way conflict and job burnout of work-families, especially for the conflict and burnout of medical staff in the context of two children.
\end{abstract}

Keywords-work-family two-way conflict; work-family conflict; family-work conflict; burnout

\section{INTRODUCTION}

With the improvement of people's basic living standards, the growth of spiritual and cultural needs, and the advent of the second child era, the two-way conflict between work-families will become more and more serious, and the high degree of burnout will become a common phenomenon. In the contemporary environment, medical staff are faced with enormous work pressures and long working hours. This highintensity work requires coordination of the coordination of family roles and job roles, resulting in the frequent occurrence of two-way conflicts in work-families, which greatly affects the performance, satisfaction and health of medical staff. It is also a soil for job burnout. The emergence and aggravation of this phenomenon will gradually lead to a decrease in work efficiency and even lead to some physical or mental illness and resignation. Therefore, it is an urgent task to study the impact of two-way conflicts of work-families on job burnout and related solutions.

Early researchers believe that the conflict between work and family is one-dimensional. With the deepening of research in this field, it is found that work and family conflicts have two dimensions, including work intervention family and family intervention work [1]. The interaction between WIF and FIW in different dimensions and the outcome variables in the field of work and family has interactive characteristics. Therefore, the two-way relationship between work-family conflicts and the interaction effects of outcome variables are worthy of further study. Burnout is a phenomenon of "emotional exhaustion, disintegration of personality and reduced sense of accomplishment when serving a professional subject" [2]. Among them, emotional exhaustion refers to enthusiasm completely lost; Personality disintegration refers to the fact that an individual faces a client or job in a negative, indifferent or extremely evasive manner, showing irritability, negativity, a lack of emotional input, etc. [3]; The low sense of achievement refers to the tendency of individuals to negatively evaluate the meaning and value of their work [4]. In the Chinese context, $\mathrm{Li}$ Chaoping and Shi Kan, who first used medical personnel as research objects, found that there was a significant correlation between work-family time conflict and emotional exhaustion, but not related to emotional alienation and personal accomplishment in a significant level, work-family conflicts and emotional exhaustion, personality disintegration, lack of personal achievement and overall burnout are related [6], and family-work conflicts can also significantly predict a reduction in personal accomplishment [8]. In summary, the survey of medical staff shows that the correlation and causal relationship between work-family conflict and job burnout are more significant. Based on this, this paper proposed that there is a positive correlation between two-way conflicts between workfamilies and job burnout, the two-way conflict between workfamilies has a significant positive predictive effect on burnout, and work-family conflicts, family-work conflicts can predict hypotheses of emotional exhaustion, cynicism, and low sense of accomplishment, respectively.

\section{RESEARCH OBJECTS AND METHODS}

\section{A. Basic situation of the research object}

The results of the sample source showed that the overall health care staff was younger. the number of medical staff over the age of 26-35 was the most, accounting for $52.2 \%$, and the number of medical staff over the age of 46 was the least, accounting for $3.9 \%$. Most of them are married people, accounting for $80.3 \%$. Among them, the number of people who have one child is the most, accounting for $74.1 \%$. Most people work for $6-10$ years, accounting for $48.9 \%$. The working years of 3-5 years accounts for $28.7 \%$. The working years are 
generally low. Their academic qualifications are mostly undergraduate, accounting for $65.7 \%$, and college education also accounts for $24.7 \%$, so the overall academic qualification is low. The sample of this study is consistent with the overall sample distribution of medical staff, with representativeness and high credibility.

\section{B. Measuring tools}

1. work-family two-way conflict questionnaire. The scale includes work-family conflicts and family-work conflicts [7].

2. Burnout questionnaire. The Chinese revised MBI-GS [5] questionnaire covers three dimensions, with fifteen questions, namely emotional exhaustion (5 questions), cynicism (4 questions), and low sense of accomplishment (6 questions).

\section{Questionnaire survey and data processing}

A total of 200 field questionnaires were distributed and 178 valid questionnaires, with an effective rate of $89 \%$. The reliability and validity of the questionnaire are tested by SPSS22.0, which are higher than the test standard, indicating that the data of the scale is trustworthy. Based on this, the data is deeply analyzed.

\section{EMPIRICAL RESEARCH AND RESULTS ANALYSIS}

\section{A. Differences in demographic variables}

Medical staff have a more obvious two-way conflict between work and family and burnout. There were no significant differences in the overall and dimensions of gender, post, marriage and work-family two-way conflicts and burnout.

Different academic qualifications have significant differences in the dimensions of burnout and emotional exhaustion; different work departments have significant differences in cynicism and work-family conflict dimensions; the number of different children has significant differences in the two-way conflicting dimensions of work-families. Specifically, education has a negative correlation with emotional exhaustion and burnout. The degree of cynicism and work-family conflict in the emergency department and the internal surgery room are relatively high, while the women and children and other departments are relatively low. The greater the number of children, the higher the level of conflict between work-families.

TABLE I. STATISTICAL ANALYSIS OF VARIANCE ANALYSIS OF WORKFAMILY CONFLICTS AND BURNOUTS

\begin{tabular}{cclll}
\hline VD & SI & ED & WD & NC \\
\hline \multirow{2}{*}{ JB } & F & 3.154 & 1.793 & 0.997 \\
& Sig & 0.045 & 0.150 & 0.371 \\
\hline \multirow{2}{*}{ EO } & F & 3.075 & 2.393 & 0.277 \\
& Sig & 0.049 & 0.070 & 0.758 \\
\hline \multirow{2}{*}{ CY } & F & 0.427 & 3.069 & 1.476 \\
& Sig & 0.653 & 0.029 & 0.231 \\
\hline \multirow{2}{*}{ TWIF } & F & 0.552 & 1.429 & 3.227 \\
& Sig & 0.577 & 0.236 & 0.041 \\
\hline \multirow{2}{*}{ WIF } & F & 2.056 & 2.721 & 1.312 \\
& Sig & 0.131 & 0.046 & 0.272 \\
\hline
\end{tabular}

VD is a variable, SI is a statistical item, ED is a degree, WD is a department, and NC is the number of children.

\section{B. Relevant relationship}

There is a significant positive correlation between the twoway conflict between work-families and job burnout, which is consistent with the assumptions. Among them, the correlation of two-way conflict between work-families with the low sense of accomplishment and cynicism are more positive than with emotional exhaustion.

Compared with FWC, the positive relationship between WFC and three dimensions of job burnout is stronger, because the intervention of medical staff to the family is greater than the interference of family work.

TABLE II. CORRELATION ANALYSIS BETWEEN WORK-FAMILY CONFLICT AND JOB BURNOUT

\begin{tabular}{lcll}
\hline VD & TWIF & WIF & FIW \\
\hline JB & $0.611^{* *}$ & $0.652^{* *}$ & $0.243^{* *}$ \\
Sig & 0.000 & 0.000 & 0.001 \\
\hline LA & $0.497^{* *}$ & $0.526^{*}$ & $0.168^{*}$ \\
Sig & 0.000 & 0.000 & 0.025 \\
\hline EO & $0.216^{* *}$ & $0.287^{* *}$ & 0.116 \\
Sig & 0.004 & 0.000 & 0.124 \\
\hline CY & $0.343^{* *}$ & $0.300^{* *}$ & $0.250^{* *}$ \\
Sig & 0.000 & 0.000 & 0.001 \\
\hline **. At the 0.01 level (two-tailed), the correlation is significant; * at the
\end{tabular}
0.05 level (two-tailed), the correlation is significant.

\section{Causal relationship}

TABLE III. REGRESSION MODEL TABLE OF TWO-WAY CONFLICTS OF WORK-FAMILIES ON BURNOUT

\begin{tabular}{|c|c|c|c|c|c|c|c|}
\hline DV & IV & $\mathrm{R}^{2}$ & $\underset{2}{\mathrm{ADJR}}$ & $\mathrm{F}$ & $\beta$ & $\mathrm{t}$ & sig \\
\hline $\mathrm{JB}$ & TWIF & 0.373 & 0.370 & 104.895 & 0.661 & 10.24 & 0.000 \\
\hline \multirow{2}{*}{ JB } & WIF & \multirow{2}{*}{0.454} & \multirow{2}{*}{0.447} & \multirow{2}{*}{72.614} & 0.634 & 11.34 & 0.000 \\
\hline & FIW & & & & 0.228 & 4.084 & 0.000 \\
\hline
\end{tabular}

DV is the dependent variable, IV is the independent variable, and ADJR2 is the adjusted R2. The table below is the same as above.

Burnout can be used as one of the predictive indicators of two-way conflicts between work and family, and is consistent with assumptions. According to the model, the standardized regression equation of two-way conflict and job burnout of working families can be obtained:

Burnout $=0.661 *$ work-family two-way conflict

Burnout $=0.634 *$ work - family conflict $+0.228 *$ family work conflict 
TABLE IV. REGRESSION MODEL TABLE OF DIMENSIONS OF OCCUPATIONAL BURNOUT IN EACH DIMENSION OF TWO-WAY CONFLICTS OF WORK-FAMILIES

\begin{tabular}{|c|c|c|c|c|c|c|c|c|}
\hline Model & DV & IV & $\mathrm{R}^{2}$ & $\underset{2}{\mathrm{ADJR}}$ & $\mathrm{F}$ & $\beta$ & $\mathrm{t}$ & sig \\
\hline \multirow{2}{*}{1} & \multirow{2}{*}{ EO } & WIF & \multirow{2}{*}{0.068} & \multirow{2}{*}{0.057} & \multirow{2}{*}{6.372} & 0.256 & 3.508 & 0.001 \\
\hline & & FIW & & & & 0.048 & 0.659 & 0.511 \\
\hline \multirow{2}{*}{2} & \multirow{2}{*}{$\mathrm{CY}$} & WIF & \multirow{2}{*}{0.095} & \multirow{2}{*}{0.085} & \multirow{2}{*}{9.201} & 0.218 & 3.033 & 0.003 \\
\hline & & FIW & & & & 0.218 & 3.033 & 0.003 \\
\hline \multirow{2}{*}{3} & \multirow{2}{*}{ LA } & WIF & \multirow{2}{*}{0.352} & \multirow{2}{*}{0.344} & \multirow{2}{*}{47.50} & 0.579 & 9.508 & 0.000 \\
\hline & & FIW & & & & 0.130 & 2.144 & 0.033 \\
\hline
\end{tabular}

The regressive significance of the dimensions of the twoway conflict of work family to the dimensions of job burnout is less than 0.05 , indicating that the dimensions of the two-way conflict of working families have significant effects on burnout Among them, the sig value of family-work conflict for emotional exhaustion is greater than 0.05, which is not significant, can not explain burnout, can not be used as a predictive indicator and contradicts to the hypothesis. The dimensions of work-family conflict has $5.7 \%, 8.5 \%$, and $33.4 \%$ of the explanatory power of burnout, so burnout can use the dimensions of two-way conflict of work-families as predictive indicators, indicating that the two dimensions of the two-way conflict of working families also have a significant impact on the sense of accomplishment. According to the model results, the standardized regression equations of the dimensions of the two-way conflicts of work-families and the dimensions of job burnout are:

\section{Emotional exhaustion $=0.256^{*}$ work-family conflict}

Cynical $=0.218 *$ work - family conflict $+0.218 *$ family work conflict 2

Low sense of accomplishment $=0.579 *$ work - family conflict $+0.130 *$ family - work conflict 3

The results show that the two-way conflict of work-families has a significant positive predictive effect on job burnout, and the positive correlation between work-family conflict and burnout is significantly higher than that of family-work conflict and burnout. This is consistent with the existing research conclusions [9][10]. However, the two-way conflict between work-families and the sense of low achievement and cynicism are more positive than their positive correlation with emotional exhaustion, which is different from the conclusions studied by previous people [11]. This is because the hospital is a secondclass hospital that has just been merged by several small health clinics. The medical staff's work treatment and working environment have improved. Medical staff have little workload. The correlation between emotional exhaustion and the two-way conflict of work-families is not so obvious. However, the medical staff's professional title and academic qualifications are low, the promotion channel is narrow, the title rating is difficult, and the difficult problems are difficult to solve, which is not very beneficial to professional development. Therefore, the state of low achievement and cynicism will be more obvious, and the two-way conflict with the work-family will be more closely linked. In general, the work-family conflicts of health care workers have a close causal relationship with job burnout. Therefore, the more serious the work-family conflicts and family-work conflicts of medical staff, the more obvious their emotional exhaustion, cynicism, low sense of accomplishment and burnout.

\section{CONCLUSION AND DISCUSSION}

There are both related and causal relationships between two-way conflicts of working families and job burnout. There are both the reasons of medical staff and the mistakes of hospital administrators. Of course, the social environment also has an impact on it. Therefore, this paper will propose management suggestions from the three aspects of medical staff, hospital administrators and society. It is hoped that through the joint efforts of the three, the goal of alleviating the two-way conflict and job burnout of the work-families of medical staff can be achieved.

\section{A. Advice for health care workers}

a) Maintain an optimistic attitude to relieve emotional exhaustion. Health care workers need to constantly adjust themselves and comfort themselves, continue to maintain their positive and optimistic attitude, and reduce the impact of emotional exhaustion.

b) Improve yourself in a comprehensive way and ease the sense of accomplishment and cynicism. Health care professionals must constantly enhance their professional knowledge and practical ability, experience the sense of value and accomplishment of work, consolidate the pride of their work, and reduce the sense of accomplishment and cynicism.

c) Develop a time plan to reduce work-family conflicts. With the opening of the second-child policy, the number of patients (especially pediatrics) and the work of medical staff and family time conflicts are intensified. Therefore, medical staff should arrange and flexibly adjust the work-family time and reserve enough time for possible conflicts.

\section{B. Advice for hospital administrators}

a) Flexible scheduling to reduce work-family conflicts. Based on age, marriage and childbirth status, etc, a reasonable work scheduling plan should be established. A scientific and effective scheduling program should be developed to reduce work-family conflicts. In particular, a more flexible time management system for medical staff under the influence of the second child policy should be given to reduce the two-way conflict between their working and families.

b) Pay attention to communication and enhance positive emotions. The counseling room should be set up and effectively used to correct and alleviate the psychological problems of medical staff in a timely manner; the employee communication channel should be sound, the all-round communication platform should be established, and employees' emotional venting and problem communication should be given adequate exports; all kinds of related publicity or group building activities should be held regularly to guide the positive emotions of medical staff.

c) Regular training to improve your sense of accomplishment and responsibility. Regular training in thoughts and skills should be given to stimulate the enthusiasm of medical staff to improve the professional ability 
and skills of medical staff, foster a sense of achievement in medical staff, meet the self-value realization needs of the work, and relieve cynicism and low sense of accomplishment.

d) Appropriate incentives to compensate for workfamily conflicts. Year-end awards and various flexible benefits should be set to meet the needs of medical staff to compensate for job burnout caused by work-family conflicts. Policies should be given for additional subsidies or benefits for relatively busy departments such as emergency and internal surgery, and price compensation should be used to reduce the impact of work-family conflict on burnout.

\section{Advice to society}

Guide the positive reports of public opinion. At present, the reports on the contradiction between doctors and patients in the society are biased. Therefore, relevant government departments need to encourage the mass media to report more on the positive impact of the hospital, guide the public to learn more about medical and health related knowledge, resist false science, discrimination and other misconceptions, strengthen the care and understanding of medical staff, thereby reducing the burnout of medical staff.

\section{REFERENCES}

[1] Gutek BA, Searle S, Klepa L. Rational Versus Gender Role Explanations for Work- Family Conflict. Journal of applied psychology, 1991, 76(4): 560-568.

[2] Maslach C, Schaufeli WB, \&LeiterMP.etal.Job burnout.Annual Review Psychology, 2001, 52:397- 422.

[3] Wang Fuhua, Xie Wei \& Zhang Tuohong.Study on the Domestic and Foreign Burnout of Medical Staff[J].Psychological Science, 2013, 30(3):188-190.

[4] Zeng Lingjuan \& Wu Xinchun. An Overview of Foreign Job Burnout Research[J]. Journal of Shenyang Normal University, 2003, 27(1):81-84.

[5] Li Chaoping, Shi Kan, Luo Zhengxue, Yang Yue \& Li Li. The Relationship between Work Family Conflict and Job Burnout of Medical Staff[J]. Chinese Journal of Mental Health, 2003, 17(12):807-809.

[6] Ya-Xuan Fang. Burnout and work-family conflict among nurses during the preparation for reevaluation of a grade $A$ tertiary hospital[J].Chinese Nursing Research, 2017(4), 51-55.

[7] Netemeyer, R. G., J. S. Boles, R. Mc Murrian. Development and Alidation of Work-family Conflict and Family-work Conflict Scales. Journal of Applied Psychology, 1996, (91):400-410.

[8] Tang Hanying, Ma Hongyu, Wang Maoyun \& Wang Bin. Study on the relationship between clinician family conflict and job burnout[J].Chinese Journal of Clinical Psychology, 2010, 18(2):219-222.

[9] Wei Ling. Study on the status quo of clinician work family conflict[J]. Journal of Nanjing Medical University, 2012(6):427-430.

[10] Lu Jiafang, Shi Kan, John J. Lawler. Preliminary Study on Work-Family Conflicts[J]. Applied Psychology, 2002, 8(2): 45-50.

[11] Zhong Xiaoqin, Tian Jishu, Yan Li, Chen Qiong, Liang Xiaohong, Tang Yong, Pan Wenfen, Tang Ping \& Ma Shirong. Research on the relationship between nurses' work-family conflict and job burnout and job performance[J].Journal of Nursing Science, 2009, 24(19) 